Article

\title{
Analytical Method to Estimate the Complex Permittivity of Oil Samples
}

\author{
Lijuan $\mathrm{Su}^{1}{ }^{1}$, Javier Mata-Contreras ${ }^{1}$, Paris Vélez ${ }^{1, *(1)}$, Armando Fernández-Prieto ${ }^{2}$ (1) \\ and Ferran Martín ${ }^{1}$ \\ 1 CIMITEC, Departament d'Enginyeria Electrònica, Universitat Autònoma de Barcelona, Bellaterra, \\ 08193 Cerdanyola del Valldès, Spain; sulijuan1020@163.com (L.S.); FranciscoJavier.Mata@uab.cat (J.M.-C.); \\ ferran.martin@uab.cat (F.M.) \\ 2 Departamento de Electrónica y Electromagnetismo, Universidad de Sevilla, Av. Reina Mercedes s/n, \\ 41012 Sevilla, Spain; armandof@us.es \\ * Correspondence: Paris.Velez@uab.cat; Tel.: +34-93-581-3522
}

Received: 2 February 2018; Accepted: 23 March 2018; Published: 26 March 2018

\begin{abstract}
In this paper, an analytical method to estimate the complex dielectric constant of liquids is presented. The method is based on the measurement of the transmission coefficient in an embedded microstrip line loaded with a complementary split ring resonator (CSRR), which is etched in the ground plane. From this response, the dielectric constant and loss tangent of the liquid under test (LUT) can be extracted, provided that the CSRR is surrounded by such LUT, and the liquid level extends beyond the region where the electromagnetic fields generated by the CSRR are present. For that purpose, a liquid container acting as a pool is added to the structure. The main advantage of this method, which is validated from the measurement of the complex dielectric constant of olive and castor oil, is that reference samples for calibration are not required.
\end{abstract}

Keywords: complex dielectric constant; complex permittivity measurements; microwave sensors; complementary split ring resonator (CSRR); microstrip technology

\section{Introduction}

This paper deals with microwave sensors based on transmission lines loaded with resonant elements. Within this topic, split ring resonators (SRRs) [1] and their complementary counterparts, complementary split ring resonators (CSRRs) [2], have been extensively used as sensing elements due to the high sensitivity of their electromagnetic properties to the surrounding medium [3-34]. Three main sensing strategies in SRR or CSRR-loaded lines have been considered: (i) variation in the notch frequency and depth caused by resonator loading (including dielectric loading) [3-15]; (ii) frequency splitting in transmission lines loaded with pairs of resonant elements, which is caused by asymmetric dielectric loading [16-23]; and (iii) coupling modulation sensors, where the notch depth is controlled by symmetry disruption [24-34].

Coupling modulation sensors are based on symmetry properties. Such sensors are implemented by symmetrically loading a host line with a symmetric resonator. The line and resonant element must be selected such that in the unperturbed state (perfect symmetry), line-to-resonator coupling is prevented. This is achieved if the symmetry planes of the line and resonator are of a different electromagnetic sort, i.e., one of them is a magnetic wall, and the other one is an electric wall. For instance, if the host line is implemented in coplanar waveguide (CPW) technology, where the line axis (symmetry plane) is a magnetic wall for the fundamental CPW mode, a convenient resonator is the SRR, since it exhibits an electric wall (symmetry plane) at the fundamental resonance frequency [24]. Coupling modulation 
sensors have been mainly applied to the measurement of spatial variables and velocities by etching the resonant element in a movable substrate, with regard to the one of the host line [27-34].

In frequency-splitting sensors, a transmission line (or a more complex, but symmetric, structure) is symmetrically loaded with a pair of (not necessarily symmetric) resonators. In the unperturbed state (i.e., by preserving the symmetry), a single notch in the transmission coefficient appears. However, if symmetry is disrupted, e.g., by means of an asymmetric dielectric loading, then two notches arise, and the distance between the notches is related to the level of asymmetry. This type of sensor has been used for the measurement of the complex dielectric constant of liquids, particularly mixtures of Deionized (DI) water and ethanol [23]. Reference liquids with well-known dielectric constant are needed for calibration purposes, and the dielectric properties of the liquid under test (LUT) are obtained from the frequency distance and the difference in notch depth between the two resonances (notches) that is generated when the reference liquid and the LUT are different (producing an asymmetry).

The most simple and extended microwave sensors implemented by means of resonator-loaded lines are those based on the resonance frequency and notch depth variation. In this case, a single resonator electromagnetically coupled to the line is needed, and no further requirements relative to symmetry apply. Any kind of perturbation in the surrounding medium of the resonant element that is able to alter the resonance frequency and/or notch depth is susceptible to be sensed. As compared to the previous sensors (frequency-splitting and coupling modulation sensors), these sensors are less immune to cross-sensitivities (e.g., those caused by ambient factors such as moisture or temperature), and hence are less robust against the effects of environmental changes. Under some circumstances, this is not an issue. Nevertheless, these sensors in general need calibration, using reference samples with well-known dielectric properties, provided that the application is focused on the measurement of the complex dielectric constant of materials.

In this paper, we present a sensor based on the variation of the notch depth and frequency of a resonator-loaded line, which is useful for the measurement of the complex dielectric constant of materials. Although this strategy for sensing (based on notch depth and frequency variation) is not new, the sensing approach for determining the complex dielectric constant is unconventional, since it is based on an analytical method, and calibration is not needed. However, certain requirements related to the characteristics of the line and material under characterization (to be discussed) must be satisfied. The sensing strategy is based on the analysis carried out by Su et al. in [13], and the method was first applied by Su et al. in [14] for the estimation of the complex dielectric constant of liquids (DI water). In this paper, a novel structure that is specifically suited to determine the dielectric constant and loss tangent of oil samples at the S-band is reported. Additionally, the analytical sensing method is described in detail, and its limitations are discussed.

\section{Description of the Proposed Sensor, Working Principle, and Sensing Method}

The proposed sensing structure is depicted in Figure 1. It consists of an embedded microstrip line loaded with a square-shaped complementary split ring resonator (CSRR), which acts as the sensing element. The main advantage of using a CSRR is that this resonator exhibits a distributed capacitance along the whole perimeter, and for this reason, its capacitance is quite sensitive to the presence of a LUT on top of it. In other words, the notch frequency is quite sensitive to the permittivity of the LUT. Moreover, a liquid container is added to the ground plane surrounding the CSRR. By introducing the LUT in such a container, it is possible to achieve certain liquid depth, which is necessary in the proposed sensing method (particularly, the depth of the container is achieved if it is completely full with the LUT). Indeed, the validity of the method is based on the homogeneity of the dielectric surrounding the metallic strip of the line, and also on the homogeneity of the dielectric material (liquid in our case) surrounding the CSRR. Obviously, such homogeneity is necessary only in the regions where the electromagnetic fields generated by the line and CSRR are present. For this reason, the considered line is an (unusual) embedded microstrip line. The thickness of the dielectric layer on top of the line, $h_{2}$, which is indicated in the caption of Figure 1 , is enough to ensure that the field lines 
do not reach the air/dielectric interface. The height of the liquid pool, $h_{3}$, which is also indicated in the caption of Figure 1, has been chosen to guarantee the uniformity of the material (LUT) on top of the CSRR (a minimum LUT depth is necessary so as to ensure that the electric field lines generated in the slots of the CSRR do not cross the air/LUT interface).

The working principle of the proposed sensor is based on the variation experienced by the notch position and depth in the transmission coefficient when the LUT is introduced in the container. The presence of the liquid modifies (increases) the capacitance of the CSRR (through the effects of its dielectric constant, $\varepsilon_{L U T}$ ), and consequently, the resonance frequency of this element shifts down. On the other hand, the notch depth is intimately related to losses, and such losses are influenced by the loss tangent $\left(\tan \delta_{L U T}\right)$ of the LUT. Therefore, the determination of such a loss parameter $\left(\tan \delta_{L U T}\right)$ is possible. Obviously, by obtaining the variation of the notch frequency and depth of liquid samples with well-known complex permittivity, calibration curves can be generated, and the measurement of the dielectric constant and loss tangent of the LUT can be achieved [23]. In this paper, we propose an unconventional method in which the measurement of such material parameters does not require any calibration (as long as the measurement of the dielectric constant and loss tangent of the LUT is done from an analytical method). However, the complex permittivity of the substrate material must be accurately known, as usual in low-loss commercial microwave substrates. Note that the use of low-loss substrates (i.e., with a small loss tangent) is necessary, especially if the materials under test exhibit moderate or low losses. Otherwise, substrate losses may degrade the sensitivity of the method for the measurement of material losses (LUT in our case).

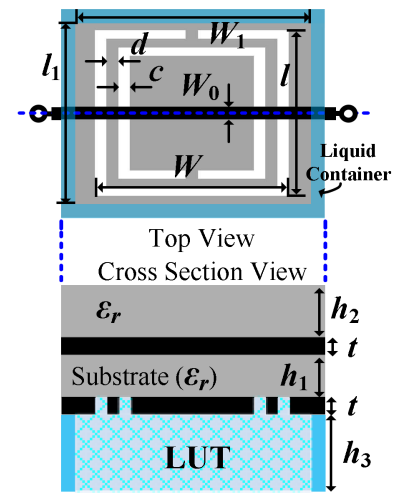

(a)

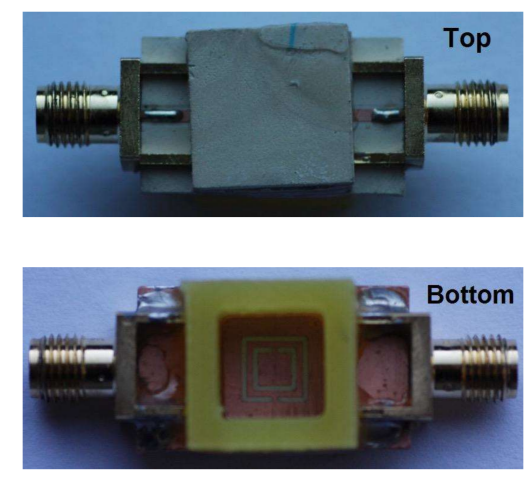

(b)

Figure 1. Top and cross-section views (a) and photograph (b) of the complementary split ring resonator (CSRR)-loaded embedded microstrip line. Dimensions are: $W=l=5.92 \mathrm{~mm}, c=d=0.5 \mathrm{~mm}$, $W_{0}=1.15 \mathrm{~mm}, W_{1}=l_{1}=9.92 \mathrm{~mm}, h_{1}=1.27 \mathrm{~mm}, h_{2}=3.81 \mathrm{~mm}, h_{3}=7.2 \mathrm{~mm}$, and $t=35 \mu \mathrm{m}$. The substrate is Rogers RO3010 with dielectric constant $\varepsilon_{r}=10.2$, and the container is FR4.

The sensing method, which will be discussed next, is based on the lumped element equivalent circuit model of the structure of Figure 1, which is depicted in Figure 2. Concerning the reactive elements of the model, $L_{c}$ and $C_{c}$ are the inductance and capacitance, respectively, of the CSRR, $L$ models the inductance of the conductive strip (line), and $C$ accounts for the capacitance between such strip and the inner region of the CSRR. Losses include substrate losses, through $R_{s}$, plus CSRR losses, modeled by $R_{M}$ (ohmic losses) and $R_{D}$ (dielectric losses of the substrate). Radiation losses are excluded, since electromagnetic simulations of the structure by excluding dielectric and ohmic losses (not shown) indicate that unitarity is preserved in the region of interest. The sensing method is based on the following equations [11]:

$$
R_{e q}=\frac{1}{R_{S} C^{2} \omega^{2}}+\frac{R_{M} R_{D}^{2}+R_{D} L_{c}^{2} \omega^{2}}{R_{D}^{2}\left(1-L_{c} C_{c} \omega^{2}\right)^{2}+\left(L_{c} \omega+R_{M} R_{D} C_{c} \omega\right)^{2}}
$$




$$
\chi_{e q}=\frac{-1}{C \omega}+\frac{R_{D}^{2} L_{c} \omega\left(1-L_{c} C_{c} \omega^{2}\right)-R_{M} R_{D}\left(L_{c} \omega+R_{M} R_{D} C_{c} \omega\right)}{R_{D}^{2}\left(1-L_{c} C_{c} \omega^{2}\right)^{2}+\left(L_{c} \omega+R_{M} R_{D} C_{c} \omega\right)^{2}}
$$

where $\omega$ is the angular frequency. The previous equations provide the real (1) and imaginary (2) part of the impedance of the shunt branch. Another necessary equation in this analytical method is the expression providing the magnitude of the transmission coefficient at the frequency, $\omega_{0}$, where $\chi_{e q}=0$, that is, [11]:

$$
\left|S_{21}\right|_{\omega_{0}}=\frac{2 Z_{0} R_{e q}}{\sqrt{\left(2 Z_{0} R_{e q}+Z_{0}^{2}-L^{2} \omega^{2}\right)^{2}+\left[2 L \omega\left(Z_{0}+R_{e q}\right)\right]^{2}}}
$$

with $Z_{0}$ being the reference impedance of the ports.

Let us consider the structure of Figure 1 without LUT in the container (unloaded CSRR). From the measured frequency response, the reactive parameters $\left(L_{c}, C_{c}, L\right.$, and $\left.C\right)$ can be inferred using the parameter extraction method reported by Bonache et al. in [35]. On the other hand, due to the uniformity of the dielectric material surrounding the strip line (with well-known loss parameter, $\tan \delta$ ), $R_{S}$ and $R_{D}$ can be obtained by means of [11]:

$$
\begin{gathered}
\tan \delta=\frac{1}{Q_{s}}=\frac{1}{R_{S} C \omega} \\
\tan \delta=\frac{1+\varepsilon_{r}}{R_{D} C_{c} \varepsilon_{r} \omega}
\end{gathered}
$$

where $Q_{s}$ is the substrate quality factor, and $\varepsilon_{r}$ is the dielectric constant of the substrate. Equation (4) is strictly valid in an embedded microstrip line, assuming that slots are not present in the ground plane. In our case, this condition is not satisfied due to the presence of the CSRR. However, as long as the side dimension of the CSRR, $l$, is high compared to $c$ and $d$, Expression (4) provides a good approximation of the loss factor of the substrate. Expression (5), on the other hand, is derived as follows. The capacitance of the unloaded CSRR (i.e., without LUT) is given by the contribution of the substrate, $C_{C_{-} \text {subs }}$, plus the contribution of the air region, $C_{c_{-} \text {air }}$, (see Figure $3 a$ ) i.e.:

$$
C_{c}=C_{c_{-} \text {subs }}+C_{c_{-} \text {air }}
$$

Since the capacitance of the air region is related to the capacitance of the substrate by $C_{C_{-} \text {subs }}=\varepsilon_{r} \cdot C_{c_{-} a \text { air }}$, it follows that:

$$
\varepsilon_{r} C_{c}=C_{c_{-} \text {subs }}\left(1+\varepsilon_{r}\right)
$$

The loss tangent of the substrate can be also expressed as:

$$
\tan \delta=\frac{1}{R_{D} C_{C_{-} \text {subs }} \omega}
$$

where it is assumed that the field lines do not cross the air/substrate interface of the CSRR slots, and the metal thickness is neglected. The actual metal thickness is $t=35 \mu \mathrm{m}$, as indicated in the caption of Figure 1, but this thickness is small enough, and hence, the above assumption is justified. Note also that $R_{D}=R_{D_{-} \text {subs }}$ (provided air can be considered as a perfect isolator, with $R_{D_{-} \text {air }}=\infty$ ), and for this reason, $R_{D_{-} s u b s}$ in the denominator of Equation (8) can be replaced by $R_{D}$, as indicated. By isolating $C_{C_{-} \text {subs }}$ in Equation (7) and introducing the resulting expression in Equation (8), Equation (5) is finally obtained. Thus, from equations (4) and (5), the dielectric loss parameters $R s$ and $R_{D}$ can be obtained. Finally, the ohmic resistance $R_{M}$ is obtained from the measurement of the transmission coefficient at $\omega_{0}$, which is also given by Equation (3). From Equation (3), the real part of the shunt impedance, $R_{\text {eq }}$, can be obtained, and from it, $R_{M}$ can be inferred using Equation (1).

Once $R_{M}$ is known, by loading the container with the LUT, all of the model parameters remain invariable except $C_{c}$ and $R_{D}$, which are influenced by the properties of LUT. Let us consider that $C_{c}$ is 
the CSRR capacitance with the container empty (as defined before), and $C_{c}{ }^{\prime}$ is the CSRR capacitance with the presence of the LUT. The notch (angular) frequencies in both cases are given by:

$$
\begin{gathered}
\omega_{0}=\frac{1}{\sqrt{L_{c}\left(C+C_{c}\right)}} \\
\omega_{0}^{\prime}=\frac{1}{\sqrt{L_{c}\left(C+C_{c^{\prime}}\right)}}
\end{gathered}
$$

The capacitance $C_{c}{ }^{\prime}$ is given by:

$$
C_{c^{\prime}}=C_{C_{-} s u b s}+C_{C_{-} L U T}=C_{C_{-} s u b s}+\frac{\varepsilon_{L U T}}{\varepsilon_{r}} C_{C_{-} s u b s}
$$

where $\varepsilon_{L U T}$ is the dielectric constant of the LUT. With equations (7) and (11), $C_{c}{ }^{\prime}$ can be expressed in terms of $C_{c}$ as follows:

$$
C_{c^{\prime}}=C_{c}\left(\frac{\varepsilon_{r}+\varepsilon_{L U T}}{\varepsilon_{r}+1}\right)
$$

By introducing Equation (12) in (10), the dielectric constant of the LUT can be isolated, resulting in the following expression:

$$
\varepsilon_{L U T}=1+\frac{\left(\omega \prime_{0}^{-2}-\omega_{0}^{-2}\right)}{L_{c} C_{c}}\left(1+\varepsilon_{r}\right)
$$

Therefore, from the measurement of the notch frequencies for the empty and full container, the dielectric constant of the LUT can be inferred using Equation (13):

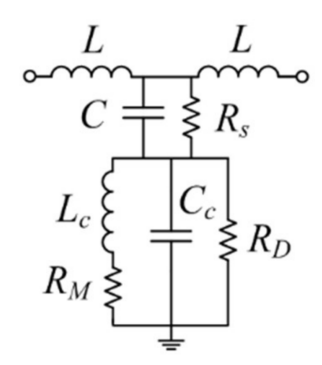

Figure 2. Lumped element equivalent circuit of the CSRR-loaded embedded microstrip line, without liquid in the container.

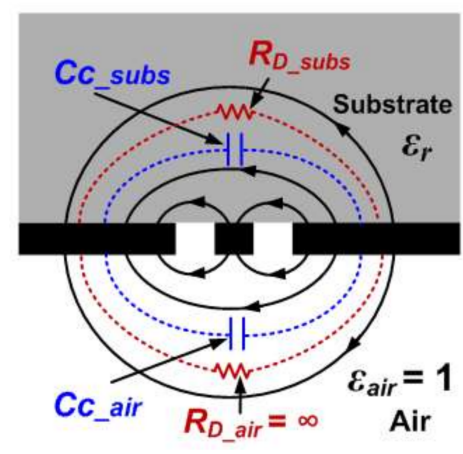

(a)

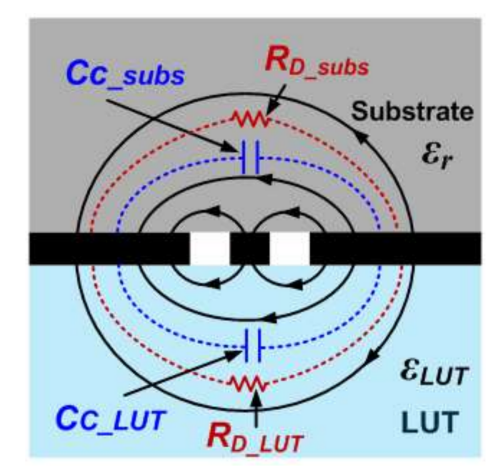

(b)

Figure 3. Cross-sectional view of the slot region of the CSRR, with electric field lines and contributions to the total CSRR capacitance and dielectric resistance. (a) Empty container; (b) full container. 
The dielectric loss of the CSRR with the presence of the LUT, $R_{D}{ }^{\prime}$, can be obtained from Equations (3) and (1), with $R_{D}$ replaced with $R_{D}{ }^{\prime}$ in Equation (1). Since $R_{D}$ is known, the loss associated to the LUT, $R_{D \_L U T}$, can be inferred, and from it, we can obtain the loss tangent of the LUT. Namely:

$$
R_{D^{\prime}}=\frac{R_{D} R_{D_{-} L U T}}{R_{D}+R_{D \_L U T}}
$$

since $R_{D}=R_{D \_s u b s}$, as indicated before. From Equation (14), $R_{D \_L U T}$ can be isolated, and once $R_{D \_L U T}$ is known, the loss tangent of the LUT can be inferred according to:

$$
\tan \delta_{L U T}=\frac{1}{R_{D_{-} L U T} C_{C_{-} L U T} \omega}
$$

with $C_{c_{-} L U T}$ given by:

$$
C_{C_{-} L U T}=C_{c^{\prime}} \frac{\varepsilon_{L U T}}{\varepsilon_{r}+\varepsilon_{L U T}}
$$

\section{Results}

The frequency response of the empty sensing structure of Figure 1 is depicted in Figure 4. This includes the measured response (inferred from the Keysight N5221A vector network analyzer), the electromagnetically simulated response (inferred from CST Microwave Studio suite 2010), and the circuit response (inferred from the element values of the circuit model, as shown in Table 1). The considered substrate, whose dimensions are indicated in the caption of Figure 1, has a dielectric constant of $\varepsilon_{r}=10.2$, and a loss tangent of $\tan \delta=0.0023$. The measured response, with a notch frequency at $f_{0}=\omega_{0} / 2 \pi=2.54 \mathrm{GHz}$ and a notch depth of $-24.4 \mathrm{~dB}$, is in very good agreement with the circuit simulation, pointing out the validity of the model (note that parameter extraction has been carried out from the experimental data).

Table 1. Circuit parameters corresponding to the empty sensing structure of Figure 1.

\begin{tabular}{cccccccc}
\hline$L(\mathrm{nH})$ & $C(\mathrm{pF})$ & $C_{c}(\mathrm{pF})$ & $L_{c}(\mathrm{nH})$ & $C_{c_{-} \text {subs }}(\mathrm{pF})$ & $R_{s}(\Omega)$ & $R_{D}(\Omega)$ & $\mathbf{R}_{\mathrm{M}}(\Omega)$ \\
\hline 3.70 & 1.09 & 8.07 & 0.43 & 7.35 & 25,015 & 3709 & 0.0147 \\
\hline
\end{tabular}

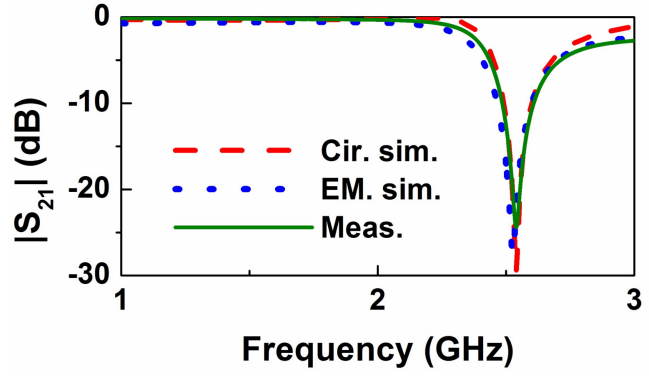

(a)

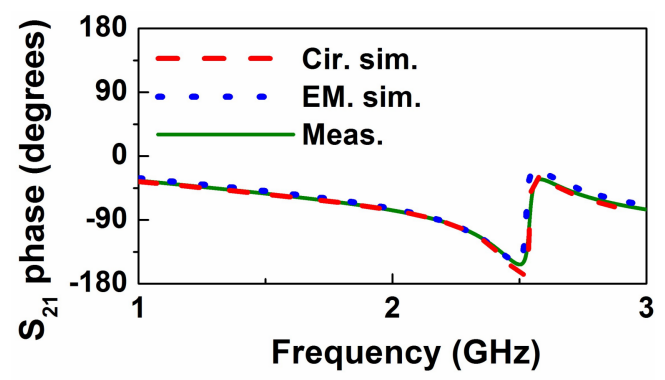

(b)

Figure 4. Transmission coefficient of the empty sensing structure of Figure 1. (a) Magnitude; (b) phase. Phase has been obtained after a reference plane shift.

By adding olive oil in the liquid container, the circuit response changes to the one indicated in Figure 5, where the notch frequency moves to $f_{0}{ }^{\prime}=\omega_{0}{ }^{\prime} / 2 \pi=2.37 \mathrm{GHz}$, and the measured notch level is found to be $-16.26 \mathrm{~dB}$. From these values, using Equation (13), the dielectric constant of the substrate is found to be $\varepsilon_{L U T}=2.93$. From equations (1) and (3), the dielectric loss of the CSRR with the presence of the LUT is found to be $R_{D}{ }^{\prime}=530.84 \Omega$, and, using Equation (14), the resulting loss associated to the oil is $R_{D \_L U T}=619.5 \Omega$. From Equation (15), using Equation (16), the loss tangent is found to be 
$\tan \delta_{L U T}=0.103$. The dielectric constant and loss tangent of olive oil obtained by the proposed method are in good agreement with independent results, which were measured with the dielectric probe kit Keysight $85070 E$ at the resonance frequency of the loaded CSRR [15], i.e., $\varepsilon_{r}=2.89$, and $\tan \delta=0.116$.

Let us briefly discuss the sources of errors in the previous results of $\varepsilon_{L U T}$ and $\tan \delta_{L U T}$. On the one hand, the implementation of the embedded microstrip line in our in-house fabrication system has been done by attaching two commercially available substrates. This may somehow alter the uniformity of the substrate, as required. On the other hand, for the validity of Equation (4), the side dimension of the CSRR must be large compared to the width of the microstrip line and the thickness of the dielectric layer between the strip line and the ground plane. This condition is satisfied in our case to a good approximation, but some source of error in the determination of $R_{S}$ is expected. Nevertheless, these error sources are difficult to estimate.

Another error source comes from the tolerance in the value of the dielectric constant of the considered substrate, i.e., $\varepsilon_{r}=10.2 \pm 0.3$ (or $\Delta \varepsilon_{r}= \pm 0.3$ ), according to the data sheet (the error in the loss tangent is not given, and therefore we will assume that this value, 0.0023 , is accurate enough so as to neglect its uncertainty). The effects of $\Delta \varepsilon_{r}$ on $\varepsilon_{L U T}$ can be inferred from Equation (13), where it can be seen that the corresponding error is simply:

$$
\Delta \varepsilon_{L U T}=\frac{\left(\omega \prime_{0}^{-2}-\omega_{0}^{-2}\right)}{L_{c} C_{c}} \Delta \varepsilon_{r}
$$

and the evaluation of Equation (17) gives $\Delta \varepsilon_{L U T}= \pm 0.05$. Note also that the error in the dielectric constant of the substrate material influences $R_{D}$ (see Equation (5)). Replacing $\varepsilon_{r}$ with $\varepsilon_{r} \pm \Delta \varepsilon_{r}$ in Equation (5), and assuming that $\Delta \varepsilon_{r}<<\varepsilon_{r}$, the error in $R_{D}$ can be approximated as:

$$
\Delta R_{D}=\frac{\Delta \varepsilon_{r}}{\tan \delta C_{c} \varepsilon_{r}^{2} \omega}
$$

giving $\Delta R_{D}= \pm 9.74 \Omega$. Through conveniently propagating this error in Equation (1), one obtains $R_{M}=0.0147 \pm 0.00003 \Omega$, and finally, the error in the loss tangent is found to be $\Delta \tan \delta_{L U T}= \pm 0.0065$.

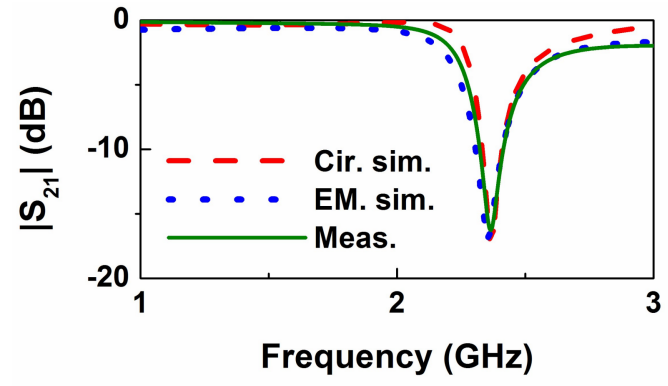

(a)

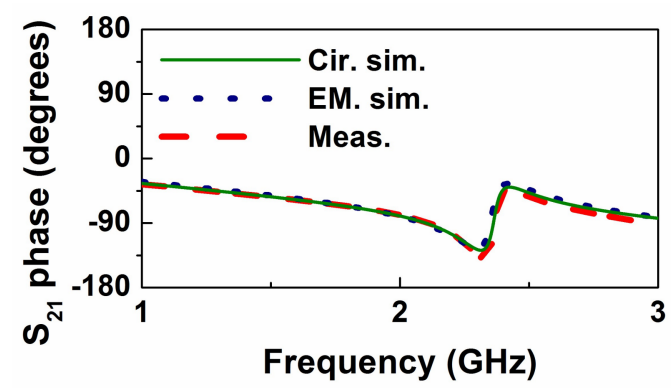

(b)

Figure 5. Transmission coefficient of the sensing structure with olive oil in the container. (a) Magnitude; (b) phase. Phase has been obtained after the reference plane shift.

We have also considered the estimation of the complex permittivity of castor oil. The corresponding response is depicted in Figure 6, where it can be appreciated that the notch frequency shifts down to $f_{0}^{\prime}=\omega_{0}^{\prime} / 2 \pi=2.31 \mathrm{GHz}$, and the measured notch level is found to be $-13.05 \mathrm{~dB}$. With these values, the analytical expressions of the method, and the previous error analysis, the resulting values of the dielectric constant and loss tangent are found to be $\varepsilon_{L U T}=3.64 \pm 0.07$, and $\tan \delta_{L U T}=0.139 \pm 0.0060$. The values measured with the dielectric probe kit $85070 E$ [15] are in this case $\varepsilon_{r}=3.32$, and $\tan \delta=0.105$. Thus, the prediction of the method is not so good in this case, but it provides a reasonable estimation on account of the error sources mentioned (note that we have only taken into account the error generated by 
the tolerance in the dielectric constant of the substrate material, but not the other effects, which are difficult to evaluate in practice).

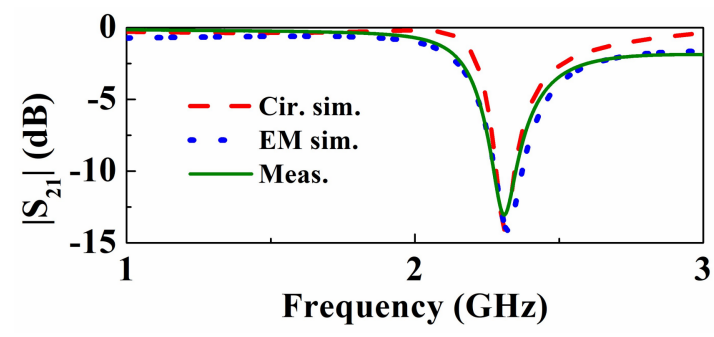

(a)

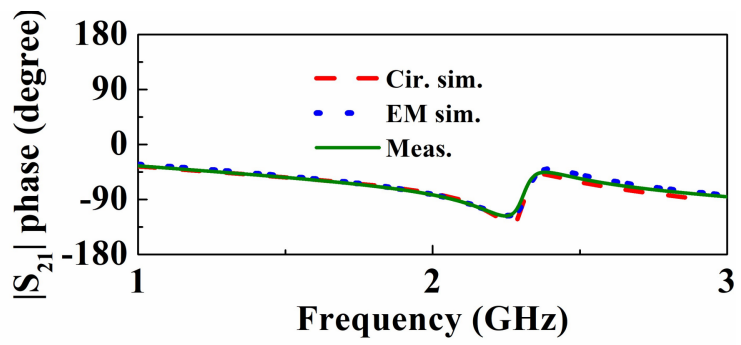

(b)

Figure 6. Transmission coefficient of the sensing structure with castor oil in the container. (a) Magnitude; (b) phase. Phase has been obtained after the reference plane shift.

\section{Discussion}

Since the proposed method is based on the variation of the notch (resonance) frequency and depth, sensor sensitivities (which are defined as the variation of the notch frequency in relation to the dielectric constant of the LUT, $\varepsilon_{L U T}$, and as the variation of the notch depth in relation to the loss tangent of the LUT, $\left.\tan \delta_{L U T}\right)$ are intimately related to the dielectric constant, $\varepsilon_{r}$, and the loss tangent, $\tan \delta$, of the substrate. This is very clear in light of Equation (13), where it can be appreciated that, for a certain value of $\varepsilon_{L U T}$, a high value $\varepsilon_{r}$ with regard to $\varepsilon_{L U T}$ should provide a small difference between the resonance frequencies $\omega_{0}$ and $\omega_{0}^{\prime}$. The dielectric constant of liquids is very diverse, from relatively small values in oil samples, to high values in DI water, for instance. Therefore, we have chosen a substrate with a moderate (with regard to the span values of liquid samples) dielectric constant of $\varepsilon_{r}=10.2$. Certainly, this is not the optimum solution for the measurement of oils. Nevertheless, the prediction of the dielectric constant in the considered oils is reasonable. Concerning the sensitivity in notch depth with loss tangent, from Equation (14), it can be seen that if substrate losses are high (i.e., small value of $R_{D}$ ), then $R_{D}{ }^{\prime}$, which is intimately related to the notch depth, will be necessarily small, and potential significant variations in $R_{L U T}$ (associated to $\tan \delta_{L U T}$, see Equation (15)) will give small variation in $R_{D}{ }^{\prime}$, and hence in the notch depth. In the considered substrate, the loss tangent is very small ( $\tan \delta=0.0023)$, giving $R_{D}=3709 \Omega$ (see Table 1 ). This loss tangent is typically two orders of magnitude smaller than those of the oil samples, and therefore, the considered substrate is appropriate for the measurement of the loss factor in such liquids.

Concerning the sensitivity of frequency with the (dimensionless) dielectric constant, this value is given in several works that are devoted to the dielectric characterization of materials; so, that comparison is feasible. The corresponding (average) values, $S_{a v}$, are given in Table 2 , where it can be appreciated that the proposed sensor exhibits a high sensitivity, which is related to a CSRR having been used as the sensing element (as discussed at the beginning of Section 2). Nevertheless, note that the value that should be compared is the one that is expressed as a percentage, i.e., $S_{a v, f}$ where the relative variation in frequency is considered. The reason is that at high frequencies, the absolute variation of the notch frequency with the dielectric constant is larger. It is remarkable that $S_{a v, f}$ is larger in the sensors of references $[15,36]$ and in this work. The reason is that the average sensitivity is taken in a different range of dielectric constants. That is, in references $[10-12,23,37,38]$, mixtures of DI water and ethanol are considered, with variations in dielectric constant between roughly 30 and 80. This high dielectric constant span explains the lower values of the sensitivity compared to those in $[15,36]$ and those in this work, where oil samples with much smaller dielectric constants are considered. Consequently, a true and reliable comparison can only be made if the samples under test are comparable. In this regard, it can be seen that the sensor presented by Kulkarni et al. in [36] exhibits the highest value of $S_{a v, f}$, but at the expense of a more complex sensing device (that uses two ring 
resonators at different metal levels). By contrast, the sensor proposed in this work is simple; it uses a single metal layer (excluding the ground plane), its size is small, and it exhibits a good sensitivity, with $S_{a v, f}=3.58 \%$. This value is larger than the one reported by Galindo-Romera et al. in [15], where the sensing device is based on a pair of split ring resonators (SRRs) loading a microstrip line etched in a single metallic layer.

Table 2. Comparison of various sensors in terms of sensitivity.

\begin{tabular}{ccccc}
\hline Reference & $f_{\mathbf{0}}(\mathbf{G H z})$ & $S_{a v}(\mathbf{M H z})$ & $S_{a v, f}(\%)$ & Measured Permittivity Range $\left.\left(\varepsilon^{\prime}\right) /\left(\varepsilon^{\prime \prime}\right)\right)$ \\
\hline$[15]$ & 1.8 & - & 3.04 & $2.45-22.52 / 0.0387-0.939$ \\
{$[10]$} & 2 & 4.76 & 0.238 & $9-79.5 / 9-10$ \\
{$[11]$} & 1.9 & 1.53 & 0.081 & $9-80 / 10-13$ \\
{$[12]$} & 3.5 & 9.16 & 0.261 & $6.5-80 / 0.1-0.4$ \\
{$[23]$} & 0.87 & 0.79 & 0.091 & $27.86-80.86 / 3.04-10$ \\
{$[36]$} & 2.5 & 8 & 0.32 & $2-76.7 / 0.06-10.27$ \\
{$[37]$} & 20 & 59.75 & 0.298 & $20-44.7 / 23.7-33.8$ \\
{$[38]$} & 2.2 & - & 5.89 & $1.88-2.12 / 0.0094-0.016$ \\
This work & 2.5 & 89.5 & 3.58 & $2.93-3.64 / 0.3-0.5$ \\
\hline
\end{tabular}

One issue that is not present in our case, since we are not considering the characterization of solid slabs, is the air gap effect [4-6]. This effect appears as long as an air gap between the sample and the sensing element (resonator) occurs, which is inevitable when a solid sample is considered and put in contact (as required) with the sensing resonator (CSRR in our case). However, with liquids, two main problems may arise: (i) the presence of bubbles; and (ii) absorption by the substrate. The first problem can be minimized by properly treating/processing the liquid (e.g., by using vacuum chambers). In general, absorption can be minimized by using low-absorption materials and/or by protecting the sensing element with a dry film [23]. The latter approach is very effective, but in our sensing method it cannot be applied, since we need a homogeneous material at both sides of the CSRR slots. Liquid absorption by the substrate depends on the viscosity of the LUT; moreover, absorption does not proceed instantaneously. In relatively diluted liquids, such as DI water or ethanol, absorption arises, and measurements must be done immediately after introducing the LUT in the container. Such a procedure was done by Su et al. in [14], where the characterization of DI water was carried out. Absorption with DI water and the considered substrate was a relatively slow process. However, it was not possible to perform reliable measurements with ethanol. In oil samples, the viscosity is high, and absorption is very slow. Therefore, the proposed analytical method is specially suited for the characterization of oils and other viscous liquids.

\section{Conclusions}

In conclusion, we have presented and discussed in detail a sensing method for the estimation of the complex dielectric constant of liquids, which is based on a CSRR-loaded embedded microstrip line. The method is based on an analytical approach, and does not require calibration. Material parameters are simply inferred from the measured frequency response of the structure loaded with the liquid under test (LUT). We have applied this method for the determination of the dielectric constant and loss tangent of oils, and the results obtained are in reasonable agreement with the results inferred from independent measurements using dielectric characterization tools. Therefore, the method has been experimentally validated. The proposed method is fast and valid for the estimation of the complex permittivity of liquids, avoiding calibration. A more accurate determination of the complex permittivity would require alternative methods based on calibration curves, similar to those reported in the recent literature. 
Acknowledgments: This work was supported by MINECO-Spain (projects TEC2013-40600-R, TEC2013-41913-P, TEC2016-75650-R), Generalitat de Catalunya (project 2014SGR-157), Spanish Junta de Andalucía (project P12-TIC-1435), Institució Catalana de Recerca i Estudis Avançats (who awarded Ferran Martín), and by FEDER funds. Lijuan Su acknowledges the China Scholarship Council (CSC) for the grant 201306950011. The authors are grateful to F.J. Herraiz-Martínez for the independent measurements of oil parameters through the dielectric probe kit.

Author Contributions: L.S. and F.M. conceived the idea and wrote the present manuscript. The simulations where carried out by L.S. and A.F.-P. The measurements were carried out by L.S., P.V., and J.M.-C. All the authors participated in discussions relative to the presented sensing system.

Conflicts of Interest: The authors declare no conflict of interest. The founding sponsors had no role in the design of the study; in the collection, analyses, or interpretation of data; in the writing of the manuscript, and in the decision to publish the results.

\section{References}

1. Pendry, J.B.; Holden, A.J.; Robbins, D.J.; Stewart, W.J. Magnetism from conductors and enhanced nonlinear phenomena. IEEE Trans. Microw. Theory Tech. 1999, 47, 2075-2084. [CrossRef]

2. Falcone, F.; Lopetegi, T.; Baena, J.D.; Marqués, R.; Martín, F.; Sorolla, M. Effective negative-epsilon stop-band microstrip lines based on complementary split ring resonators. IEEE Microw. Wireless Compon. Lett. 2004, 14, 280-282. [CrossRef]

3. Puentes, M.; Weiss, C.; Schüßler, M.; Jakoby, R. Sensor array based on split ring resonators for analysis of organic tissues. In Proceedings of the IEEE MTT-S International Microwave Symposium Digest, Baltimore, MD, USA, 5-10 June 2011.

4. Puentes, M. Planar Metamaterial Based Microwave Sensor Arrays for Biomedical Analysis and Treatment. Springer: Heidelberg, Germany, 2014; ISBN 978-3-319-06041-5.

5. Schueler, M.; Mandel, C.; Puentes, M.; Jakoby, R. Metamaterial inspired microwave sensors. IEEE Microw. Mag. 2012, 13, 57-68. [CrossRef]

6. Boybay, M.S.; Ramahi, O.M. Material characterization using complementary split-ring resonators. IEEE Trans. Instrum. Meas. 2012, 61, 3039-3046. [CrossRef]

7. Lee, C.S.; Yang, C.L. Complementary split-ring resonators for measuring dielectric constants and loss tangents. IEEE Microw. Wireless Compon. Lett. 2014, 24, 563-565. [CrossRef]

8. Yang, C.L.; Lee, C.S.; Chen, K.W.; Chen, K.Z. Noncontact measurement of complex permittivity and thickness by using planar resonators. IEEE Trans. Microw. Theory Tech. 2016, 64, 247-257. [CrossRef]

9. Abduljabar, A.A.; Rowe, D.J.; Porch, A.; Barrow, D.A. Novel microwave microfluidic sensor using a microstrip split-ring resonator. IEEE Trans. Microw. Thory Tech. 2014, 62, 679-688. [CrossRef]

10. Ebrahimi, A.; Withayachumnankul, W.; Al-Sarawi, S.; Abbott, D. High-sensitivity metamaterial-inspired sensor for microfluidic dielectric characterization. IEEE Sens. J. 2014, 14, 1345-1351. [CrossRef]

11. Withayachumnankul, W.; Jaruwongrungsee, K.; Tuantranont, A.; Fumeaux, C.; Abbott, D. Metamaterialbased microfluidic sensor for dielectric characterization. Sens. Actuators A Phys. 2013, 189, $233-237$. [CrossRef]

12. Salim, A.; Lim, S. Complementary Split-Ring Resonator-Loaded Microfluidic Ethanol Chemical Sensor. Sensors 2016, 16, 1802. [CrossRef] [PubMed]

13. Su, L.; Mata-Contreras, J.; Vélez, P.; Martín, F. Estimation of conductive losses in complementary split ring resonator (CSRR) loading an embedded microstrip line and applications. In Proceedings of the IEEE MTT-S International Microwave Symposium (IMS), Honolulu, HI, USA, 476-479 June 2017.

14. Su, L.; Mata-Contreras, J.; Vélez, P.; Martín, F. Estimation of the complex permittivity of liquids by means of complementary split ring resonator (CSRR) loaded transmission lines. In Proceedings of the IEEE MTT-S International Microwave Workshop Series on Advanced Materials and Processes (IMWS-AMP 2017), Pavia, Italy, 20-22 September 2017.

15. Galindo-Romera, G.; Herraiz-Martínez, F.J.; Gil, M.; Martínez-Martínez, J.J.; Segovia-Vargas, D. Submersible printed split-ring resonator-based sensor for thin-film detection and permittivity characterization. IEEE Sens. J. 2016, 16, 3587-3596. [CrossRef] 
16. Horestani, A.K.; Naqui, J.; Shaterian, Z.; Abbott, D.; Fumeaux, C.; Martín, F. Two-Dimensional Alignment and Displacement Sensor based on Movable Broadside-Coupled Split Ring Resonators. Sens. Actuators A Phys. 2014, 210, 18-24. [CrossRef]

17. Naqui, J.; Damm, C.; Wiens, A.; Jakoby, R.; Su, L.; Martín, F. Transmission lines loaded with pairs of magnetically coupled stepped impedance resonators (SIRs): Modeling and application to microwave sensors. In Proceedings of the IEEE MTT-S International Microwave Symposium (IMS), Tampa, FL, USA, 1-4 June 2014.

18. Su, L.; Naqui, J.; Mata-Contreras, J.; Martín, F. Modeling metamaterial transmission lines loaded with pairs of coupled split ring resonators. IEEE Ant. Wirel. Propag. Lett. 2015, 14, 68-71. [CrossRef]

19. Su, L.; Naqui, J.; Mata-Contreras, J.; Martín, F. Dual-band epsilon-negative (ENG) transmission line metamaterials based on microstrip lines loaded with pairs of coupled complementary split ring resonators (CSRRs): Modeling, analysis and applications. In Proceedings of the 9th International Congress on Advanced Electromagnetic Materials in Microwaves and Optics, Metamaterials 2015, Oxford, UK, 7-12 September 2015.

20. Su, L.; Naqui, J.; Mata-Contreras, J.; Vélez, P.; Martín, F. Transmission line metamaterials based on pairs of coupled split ring resonators (SRRs) and complementary split ring resonators (CSRR): A comparison to the light of the lumped element equivalent circuits. In Proceedings of the International Conference on Electromagnetics for Advanced Applications (ICEAA 2015), Torino, Italy, 7-11 September 2015.

21. Su, L.; Naqui, J.; Mata-Contreras, J.; Martín, F. Modeling and applications of metamaterial transmission lines loaded with pairs of coupled complementary split ring resonators (CSRRs). IEEE Ant. Wirel. Propag. Lett. 2016, 15, 154-157. [CrossRef]

22. Su, L.; Mata-Contreras, J.; Naqui, J.; Martín, F. Splitter/combiner microstrip sections loaded with pairs of complementary split ring resonators (CSRRs): Modeling and optimization for differential sensing applications. IEEE Trans. Microw. Theory Tech. 2016, 64, 4362-4370. [CrossRef]

23. Vélez, P.; Su, L.; Grenier, K.; Mata-Contreras, J.; Dubuc, D.; Martín, F. Microwave Microfluidic Sensor Based on a Microstrip Splitter/Combiner Configuration and Split Ring Resonators (SRRs) for Dielectric Characterization of Liquids. IEEE Sens. J. 2017, 17, 6589-6598. [CrossRef]

24. Naqui, J.; Durán-Sindreu, M.; Martín, F. Novel sensors based on the symmetry properties of split ring resonators (SRRs). Sensors 2011, 11, 7545-7553. [CrossRef] [PubMed]

25. Martín, F. Artificial Transmission Lines for RF and Microwave Applications. John Wiley: Hoboken, NI, USA, 2015; ISBN 9781119058403.

26. Naqui, J. Symmetry Properties in Transmission Lines Loaded with Electrically Small Resonators: Circuit Modeling and Applications; Springer: Heidelberg, Germany, 2016; ISBN 978-3-319-24566-9.

27. Naqui, J.; Durán-Sindreu, M.; Martín, F. Alignment and position sensors based on split ring resonators. Sensors 2012, 12, 11790-11797. [CrossRef]

28. Horestani, A.K.; Fumeaux, C.; Al-Sarawi, S.F.; Abbott, D. Displacement sensor based on diamond-shaped tapered split ring resonator. IEEE Sens. J. 2013, 13, 1153-1160. [CrossRef]

29. Horestani, A.K.; Abbott, D.; Fumeaux, C. Rotation sensor based on horn-shaped split ring resonator. IEEE Sens. J. 2013, 13, 3014-3015. [CrossRef]

30. Naqui, J.; Martín, F. Transmission lines loaded with bisymmetric resonators and their application to angular displacement and velocity sensors. IEEE Trans. Microw. Theory Tech. 2013, 61, 4700-4713. [CrossRef]

31. Naqui, J.; Martín, F. Angular displacement and velocity sensors based on electric-LC (ELC) loaded microstrip lines. IEEE Sens. J. 2014, 14, 939-940. [CrossRef]

32. Horestani, A.K.; Naqui, J.; Abbott, D.; Fumeaux, C.; Martín, F. Two-dimensional displacement and alignment sensor based on reflection coefficients of open microstrip lines loaded with split ring resonators. Electron. Lett. 2014, 50, 620-622. [CrossRef]

33. Naqui, J.; Martín, F. Microwave sensors based on symmetry properties of resonator-loaded transmission lines: A review. J. Sens. 2015, 2015, 1-10. [CrossRef]

34. Naqui, J.; Coromina, J.; Karami-Horestani, A.; Fumeaux, C.; Martín, F. Angular displacement and velocity sensors based on coplanar waveguides (CPWs) loaded with S-shaped split ring resonators (S-SRR). Sensors 2015, 15, 9628-9650. [CrossRef] [PubMed]

35. Bonache, J.; Gil, M.; Gil, I.; Garcia-Garcia, J.; Martin, F. On the electrical characteristics of complementary metamaterial resonators. IEEE Microw. Wireless Compon. Lett. 2006, 16, 543-545. [CrossRef] 
36. Kulkarni, S.; Joshi, M.S. Design and analysis of shielded vertically stacked ring resonator as complex permittivity sensor for petroleum oils. IEEE Trans. Microw. Theory Tech. 2015, 63, 2411-2417. [CrossRef]

37. Chretiennot, T.; Dubuc, D.; Grenier, K. A microwave and microfluidic planar resonator for efficient and accurate complex permittivity characterization of aqueous solutions. IEEE Trans. Microw. Theory Tech. 2013, 61, 972-978. [CrossRef]

38. Abdolrazzaghi, M.; Daneshmand, M.; Iyer, A.K. Strongly enhanced sensitivity in planar microwave sensors based on metamaterial coupling. IEEE Trans. Microw. Theory Tech. 2018, 1-13. [CrossRef]

(c) 2018 by the authors. Licensee MDPI, Basel, Switzerland. This article is an open access article distributed under the terms and conditions of the Creative Commons Attribution (CC BY) license (http:/ / creativecommons.org/licenses/by/4.0/). 